\title{
THE ROLE OF SHOCKS IN NGC 6240
}

\author{
William C. Keel \\ University of Alabama
}

One of the most notable results of the IRAS mission was the recognition of a class of galaxies with large bolometric luminosities, most of whose radiation emerges in the far-infrared (typically 50-100 $\mu$ ). The space density of these "IRAS galaxies" approaches that of QSOs for comparable luminosities, so that these are a significant component of the population of galaxies. The frequent occurrence of interacting and merging galaxies among these infrared-bright systems has underscored the importance of understanding how various energetic processes (active nuclei, starbursts) are related to the disturbances produced during galaxy encounters. The same material directly responsible for the signature infrared excess of these systems degrades many of the diagnostics normally useful for probing the nature of he observed activity, so that in many systems it remains unclear just what kinds of phenomena are being observed.

The case of the infrared-bright system NGC 6240 encapsulates in many ways the major issues in understanding processes in infrared-bright systems in general. Its morphology strongly suggests that this is a merger in progress, with energetic phenomena being triggered as evidenced by strong radio emission and optical emission lines as well as the strong far-infrared output.

Evidence has been produced supporting various schemes for producing the energy being radiated by this object. Early work on the optical spectrum suggested a Seyfert 2 classification, but the ionization level and spatial extent of the emission implied a more distributed source of energy. IR line studies found evidence for young supergiants, and for immense amounts of $\mathrm{H}_{2}$ excited by low-velocity shocks, which might contribute to a large enhancement in star-formation rate. Finally, the radio-continuum morphology shows compact sources possibly associated with active nuclei in both remnants of the merger.

This is one of the nearest IR-bright galaxies objects at high luminosity ( $c z=7500$ $\mathrm{km} \mathrm{s}^{-1}$ ), so that spatially resolved studies can yield further insight into which of these processes are most important - star formation, nuclear activity, or shock excitation.

\section{NEW OBSERVATIONS: SPECTROSCOPY}

The velocity field, linewidth distribution, and ionization structure of the gas in NGC 6240 has been mapped with the Dense-Pak fiber-optic array on the Kitt Peak 4-m telescope. In all, 135 spectra were obtained through an array of 2" apertures, with considerable overlap between many of the adjacent apertures produced by offsetting the telescope between the exposures. As the strongest features with $\lambda<1.1 \mu, \mathrm{H} \alpha$ and the adjacent [N II] lines were observed, along with the neighboring [O I] and [S II] features. Maps of velocity and line width were constructed by interpolating measurements from these spectra onto a regular grid, and show irregular patterns with large velocity gradients and, particularly, an extended region of large local (shear?) velocity width.

The character of the emission is also revealing. Only in a few locations do the emission lines show ratios and widths indicating normal H II regions photionized by 
young stars (Fig. 1). Most of the emission is of a LINER-like character, and has FWHM for the lines of order $500 \mathrm{~km} \mathrm{~s}^{-1}$. Such emission might in principle reflect shock
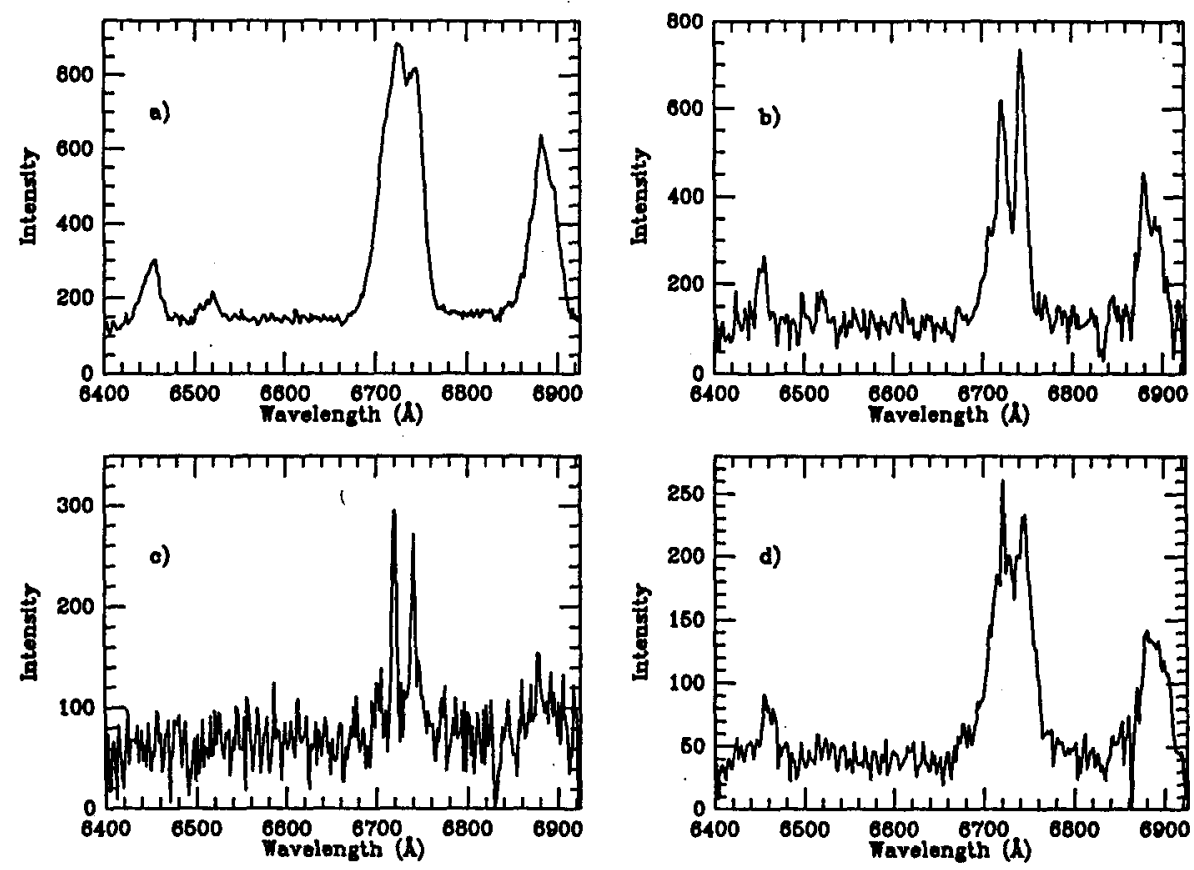

Fig. 1: Four kinds of emission spectrum in NGC 6240. a) relatively broad lines on the brighter nucleus, b) very extended LINER-line emission with linewidths $\approx 500 \mathrm{~km} \mathrm{~s}^{-1}$ FWHM, c) H II regions near the edge of the mapped region, d) very broad wings on $\mathrm{H} \alpha+[\mathrm{N} \mathrm{II}]$ suggesting reflected light from a broad-line nucleus.

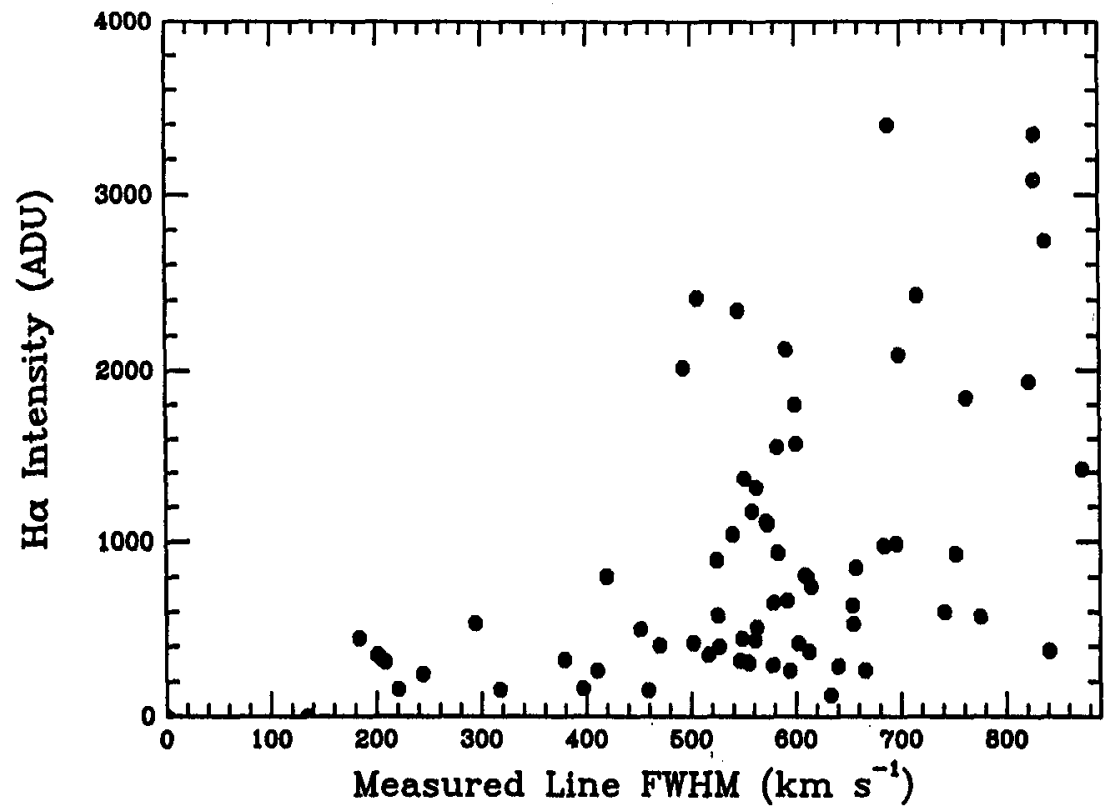

Fig. 2. Emission-line intensity versus local line width (FWHM) for NGC 6240, excluding areas within 2 arcseconds of the brighter nucleus.

excitation (at velocities of order $100 \mathrm{~km} \mathrm{~s}^{-1}$ or photoionization by a weak flat-spectrum source (such as an active nucleus). The structure of the gas provides a clue for discrim- 
inating between these possibilities - the line intensity and inferred ionization parameter do not show symmetry about the nucleus, but the line intensity is coupled to the local line width, as if the processes driving the local velocity structure are also responsible for the ionization of the gas. The relation between line intensity and emission-line FWHM is shown in Fig. 2 for all positions except the two nuclei and their immediate vicinity.

The relation between line width (dominated by local velocity structure) and maximum line intensity is a strong argument that shocks are dominant in ionizing the interstellar medium throughout the inner $5 \mathrm{kpc}$ of NGC 6240.

\section{NEW OBSERVATIONS: IMAGING}

New images, from $\mathrm{B}$ to $\mathrm{K}$ bands, have also been obtained, including a set taken with the ESO/MPI 2.2-m telescope in subarcsecond seeing. Seeing deconvolution (via a modified CLEAN algorithm) has been used to examine the two apparent nuclei and their surroundings. The two optical objects appear to be genuine stellar concentrations, with symmetric (and very compact) structures and quite similar, heavily reddened, continuum energy distributions. The latter implies that both are deeply imbedded in the extensive dust distribution of the system, and thus are close together in space as well as in projection.

Analysis of the imaging data also allows a new assessment of the contribution of reradiated starlight to the overall far-IR luminosity of the system. Considering both the strong dust lanes and the widespread diffuse dust responsible for most of the extinction, a lower limit of about $15 \%$ may be set for the contribution of a component analogous to galactic cirrus emission, in which the grains are heated by ambient visible-wavelength starlight as opposed to the UV continuum of a starburst population. As a lower limit, this calls into question the need for a strong burst of star formation on strictly energetic grounds, as has been done by Thronson et al. (1990) as well.

\section{EMISSION-LINE ENERGETICS}

The ionization of the observed gas appears to be dominated by shock heating, but closer examination is needed to assess the roles of star formation and possibly nuclear activity as well in contributing to the overall luminosity of the system. Since the emission-line luminosity is only a small fraction of the bolometric luminosity, it is not obvious that the kinetic energy of the system of gas clouds being released via collisions is sufficient to power the far-infrared excess for a significant time.

The kinetic-energy content of the gas may be expressed as

$$
E=\frac{3 m_{\mathrm{H}} D^{2}(v-\bar{v})^{2}}{2 \pi f_{i} n_{e} \alpha h \nu \mathrm{I}_{\text {line }}}
$$

where integration over the whole emission region is assumed. Here $m_{H}$ is the mass of the hydrogen atom, $\mathrm{D}$ is the system's distance, $v$ the radial velocity at each point and $\bar{v}$ the mean (the factor 3 assumes isotropic motions), $f_{i}$ is the ionized fraction of the gas by mass, $n_{e}$ the electron density, and $\mathrm{I}_{\text {line }}$ the intensity of a recombination line at frequency $\nu$ with recombination coefficient $\alpha$. The energy content of the gas in NGC 6240 may be estimated using $n_{e}$ from the [S II] $\lambda \lambda 6717,6731$ doublet ratio and direct 
integration of the intensity and velocity maps. Local gas motions (reflected in the line widths) dominate over large-scale motions (those that are spatially resolved) by about a factor 10 . For a value $f_{i}=0.01, E=2 \times 10^{56} \mathrm{erg} \mathrm{s}^{-1}$, scaling inversely as the ionized fraction. Only for $f_{i}<10^{-4}$ could cloud collisions power the total energy output of NGC 6240 in the far-infrared for the $10^{7}$ years needed for a nontrivial chance of seeing the system in such a phase. The large CO luminosity of NGC 6240 does suggest a large value of $f_{i}$, but the relation between $\mathrm{CO}$ emission and $\mathrm{H}_{2}$ mass needs to be better understood in such systems for reliable conclusions.

Particularly if most of the gas in NGC 6240 is neutral or molecular, the situation is remniscent of the proposal by Harwit et al. (1987) that collisional heating could be dominant in IR-bright galaxies. If the optical and near-IR line emission from such systems is dominated by shocked gas (as proposed here), searches for such systems via, for example, $2-4 \mu$ slitless or multiobject spectroscopy from space to search for strong $\mathrm{H}_{2}$ emission, could yield a direct tracer of the rate of disk galaxy mergers and its evolution with cosmic time.

A full presentation of the data, and details of the analysis, have been submitted to the Astronomical Journal.

This study was made possible by allocations of observing time at Kitt Peak National Observatory, the European Southern Observatory, and the Observatorio del Roque de los Muchachos, and supported in part by EPSCoR grant RII-8610699.

\section{REFERENCES}

Harwit, M., Houck, J.R., Soifer, B.T., and Palumbo, G.G.C. 1987, Astrophys. J. 315, 28.

Thronson, H. A., Jr., Majewski, S., Descartes, L., and Hereld, M. 1990, Astrophys. J. (submitted). 